\title{
A Time-Varying Non-Parametric Methodology for Assessing Changes in QT Variability Unrelated to Heart Rate Variability
}

\author{
Michele Orini*, Esther Pueyo, Pablo Laguna, Raquel Bailón
}

\begin{abstract}
Objective: To propose and test a novel methodology to measure changes in QT interval variability (QTV) unrelated to $R R$ interval variability ( RRV) in non-stationary conditions. Methods: Time-frequency coherent and residual spectra representing QTV related (QTVrRRV) and unrelated (QTVuRRV) to RRV, respectively, are estimated using time-frequency Cohen's class distributions. The proposed approach decomposes the nonstationary output spectrum of any two-input one-output model with uncorrelated inputs into two spectra representing the information related and unrelated to one of the two inputs, respectively. An algorithm to correct for the bias of the timefrequency coherence function between $Q T V$ and $R R V$ is proposed to provide accurate estimates of both QTVuRRV and QTVRRRV. Two simulation studies were conducted to assess the methodology in challenging non-stationary conditions and data recorded during head-up tilt in $\mathbf{1 6}$ healthy volunteers were analyzed.

Results: In the simulation studies, QTVuRRV changes were tracked with only a minor delay due to the filtering necessary to estimate the non-stationary spectra. The correlation coefficient between theoretical and estimated patterns was $>0.92$ even for extremely noisy recordings (SNR in QTV $=-10 \mathrm{~dB}$ ). During head-up tilt, QTVrRRV explained the largest proportion of QTV, whereas QTVuRRV showed higher relative increase than QTV or QTVrRRV in all spectral bands $(P \leq 0.05$ for most pairwise comparisons).

Conclusion: The proposed approach accurately tracks changes in QTVuRRV. Head-up tilt induced a slightly greater increase in QTVuRRV than in QTVRRRV.

Significance: The proposed index QTVuRRV may represent an indirect measure of intrinsic ventricular repolarization variability, a marker of cardiac instability associated with sympathetic ventricular modulation and sudden cardiac death.
\end{abstract}

Index Terms-Time-frequency, Spectral Coherence, Heart rate variability, QT variability, Cardiac Repolarization

\section{INTRODUCTION}

The temporal liability of ventricular repolarization is an important factor in arrhythmogenesis. Established pro-arrhythmic substrates include beat-to-beat repolarization variability [1], repolarization alternans [2], [3] and repolarization response

This work was supported by projects TIN2013-41998-R, TIN2014-53567-R and DPI2016-75458-R from Spanish Ministry of Economy and Competitiveness (MINECO), Spain, MULTITOOLS2HEART from CIBER-BBN through Instituto de Salud Carlos III, Spain, European Social Fund (EU) and Aragon Government through BSICoS group (T96) and by the European Research Council (ERC) through project ERC-2014-StG 638284.

* Corresponding Author: m.orini@ucl.ac.uk

M. Orini is with the Mechanical Engineering Department, University College London, London, UK and Barts Heart Centre at St Bartholomews Hospital, University College London, London, UK.

E. Pueyo, P. Laguna and R. Bailón are with BSICoS group, I3A, IIS Aragon, University of Zaragoza, Zaragoza, Spain, and with CIBER-BBN, Zaragoza, Spain to changes in heart rate [4]-[7]. Relevant information about the spatio-temporal distribution of ventricular repolarization can be non-invasively obtained from the analysis of the Twave in the surface ECG [8]. In particular, beat-to-beat QT interval variability (QTV) is an established marker carrying valuable information about ventricular patho-physiology [1]. Several studies have suggested that QTV is associated with sympathetic ventricular outflow and susceptibility to malignant ventricular arrhythmias [1], [9]-[13]. However, part of QTV may be explained by RR interval variability (RRV), which is related to QTV through the restitution properties [14]. In fact, QTV can be interpreted as the contribution of two components, one related to RRV (QTVrRRV) and another unrelated to RRV (QTVuRRV) and associated with intrinsic ventricular repolarization dynamics (VRD). Enhanced VRD is thought to be due to abnormal ion channels dynamics involving calcium [15] and potassium [16] regulation and their interaction with $\beta$-adrenergic (sympathetic) ventricular activity [15]-[17], and has been shown to be relevant for arrhythmogenesis [15], [16], [18]. Furthermore, the spectral content of QTVuRRV has been suggested to be a marker of ventricular sympathetic activity [19], which is also related to arrhythmic risk [10].

Previous techniques to remove the influence of RRV from QTV have mainly utilized heart rate corrections [10], [20], time-invariant multivariate autoregressive models [19]-[22] or other model-based approaches [23]. However, these methodologies are unable to track the dynamic response to physical, cognitive or autonomic challenges, which provides valuable insight into cardiac and cardiovascular regulation and the system's ability to adapt. Therefore, there is a need for a methodology that provides accurate and robust estimates of the dynamic profile of QTVuRRV. The aim of this study is to propose a novel framework for estimating QTVuRRV during non-stationary conditions, therefore providing a non-invasive assessment of VRD changes. The proposed methodology is non-parametric and is based on multivariate quadratic timefrequency $(\mathrm{TF})$ analysis. It uses the $\mathrm{TF}$ coherence function to separate the non-stationary spectrum of QTV into a spectrum carrying information related to RRV and another one carrying information unrelated to RRV, where the latter is assumed to be an approximation of intrinsic VRD. Importantly, the methodology includes an algorithm to correct for the bias of the TF coherence to provide accurate estimates of QTVuRRV. 


\section{A. General Model}

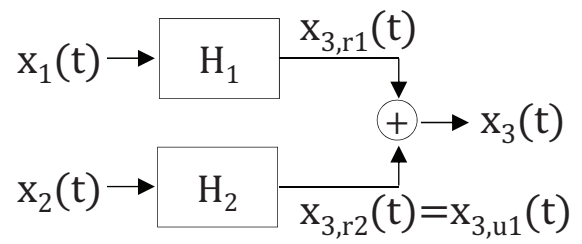

B. Calibration model for bias correction
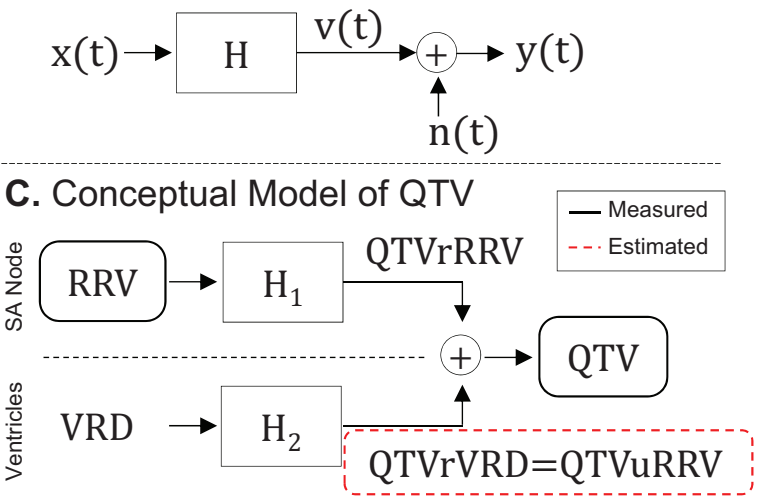

Figure 1. A: General two-input one-output model, with two uncorrelated inputs. Sub-indices $r 1$ and $r 2$ denote components of $x_{3}(t)$ related to inputs $x_{1}(t)$ and $x_{2}(t)$, respectively. Similarly, sub-index $u l$ denotes the component of $x_{3}(t)$ unrelated to inputs $x_{1}(t)$. B: Calibration model used to assess and correct the TF coherence bias. C: Physiological model of QTV adapted from (A). RRV: RR interval variability; QTV: QT interval variability; VRD: Ventricular repolarization dynamics; QTVuRRV: QTV unrelated to RRV; QTVrRRV and QTVrVRD represent QTV related to RRV and to intrinsic VRD, respectively.

\section{METHODS}

\section{A. Residual and coherent spectra}

The methodology proposed in this paper is based on classical multiple-input single-output theory [24] and applies to systems that can be interpreted as the contribution of two single-input single-output models where inputs $x_{1}(t)$ and $x_{2}(t)$ are uncorrelated random processes (see Fig. 1A). In the general time-invariant formulation, where stationarity is required, these systems can be defined as:

$$
\begin{aligned}
x_{3}(t) & =x_{3, \mathrm{r} 1}(t)+x_{3, \mathrm{u} 1}(t)=x_{3, \mathrm{r} 1}(t)+x_{3, \mathrm{r} 2}(t) \\
& =h_{1}(t) * x_{1}(t)+h_{2}(t) * x_{2}(t)
\end{aligned}
$$

where $*$ represents the convolution operator, $h_{1}(t)$ and $h_{2}(t)$ are linear impulse responses, and $x_{3, \mathrm{r} 1}(t)$ and $x_{3, \mathrm{u} 1}(t)$ represent the components of the output that are related and unrelated to $x_{1}(t)$, respectively. Note that by definition $x_{3, \mathrm{u} 1}(t)=$ $x_{3, \mathrm{r} 2}(t)$. Since inputs $x_{1}(t)$ and $x_{2}(t)$ are assumed to be uncorrelated, the auto-spectrum of the output is [24], [25]:

$$
S_{33}(f)=S_{3, \mathrm{r} 1}(f)+S_{3, \mathrm{r} 2}(f)=S_{3, \mathrm{r} 1}(f)+S_{3, \mathrm{u} 1}(f)
$$

and the spectrum of $x_{3, \mathrm{u} 1}(t)$, so called residual or conditioned spectrum [24], is:

$$
\begin{aligned}
S_{3, \mathrm{u} 1}(f) & =S_{33}(f)-S_{3, \mathrm{r} 1}(f)= \\
& =S_{33}(f)-\gamma_{31}^{2}(f) S_{33}(f)=\left[1-\gamma_{31}^{2}(f)\right] S_{33}(f)
\end{aligned}
$$

where $\gamma_{31}(f)$ is the spectral coherence between the output $x_{3}(t)$ and the input $x_{1}(t)$, defined as [26]:

$$
\gamma_{31}^{2}(f)=\frac{S_{31}^{2}(f)}{S_{11}(f) S_{33}(f)}
$$

where $S_{31}(f)$, with $\left|S_{31}(f)\right|=\left|S_{13}(f)\right|$, is the cross spectrum between $x_{1}(t)$ and $x_{3}(t)$. The spectral coherence is equal to 1 when the two processes are linearly correlated and equal to zero when they are uncorrelated. The expression $\gamma_{31}^{2}(f) S_{33}(f)$ represents the so called coherent output spectrum, i.e. the proportion of $S_{33}(f)$ coherent with the input spectrum $S_{11}(f)$. Therefore, the spectral coherence acts as a filter that decomposes the output spectrum into coherent and residual parts with respect to one of the two inputs. It is worth noting that (4) offers the possibility of estimating the coherent and residual spectra even if signals $x_{3, \mathrm{r} 1}(t)$ and $x_{3, \mathrm{u} 1}(t)$ are unknown, by processing input $x_{1}(t)$ and output $x_{3}(t)$ (Fig. 1A).

\section{B. Time-frequency approach}

Spectral analysis is extended to the TF domain by means of the quadratic Cohen's class distributions as described in previous studies [27], [28]. Briefly, auto and cross-spectra of two non-stationary signals $x(t)$ and $y(t)$ are estimated as:

$$
\begin{aligned}
& \hat{S}_{\mathrm{xy}}(t, f)=\iint_{-\infty}^{\infty} \phi_{\mathrm{d}-\mathrm{D}}(\tau, \nu) \hat{A}_{\mathrm{xy}}(\tau, \nu) e^{j 2 \pi(t \nu-f \tau)} d \nu d \tau \\
& \hat{A}_{\mathrm{xy}}(\tau, \nu)=\int_{-\infty}^{-\infty} x\left(t+\frac{\tau}{2}\right) y^{*}\left(t-\frac{\tau}{2}\right) e^{-j 2 \pi \nu t} d t
\end{aligned}
$$

where $\hat{A}_{\text {xy }}(\tau, \nu)$ is the narrow-band symmetric ambiguity function of signals $x(t)$ and $y(t)$, and $\phi_{\mathrm{d}-\mathrm{D}}(\tau, \nu)$ is an elliptical exponential kernel defined in the ambiguity function domain as [27]:

$$
\phi_{\mathrm{d}-\mathrm{D}}(\tau, \nu)=\exp \left\{-\pi\left[\left(\frac{\nu}{\nu_{0}}\right)^{2}+\left(\frac{\tau}{\tau_{0}}\right)^{2}\right]^{2}\right\}
$$

The TF coherence distribution is defined as:

$$
\hat{\gamma}_{\mathrm{xy}}^{2}(t, f)=\frac{\hat{S}_{\mathrm{xy}}^{2}(t, f)}{\hat{S}_{\mathrm{xx}}(t, f) \hat{S}_{\mathrm{yy}}(t, f)}
$$

The low-pass filtering performed by the kernel in (8) is necessary to remove the interference terms that characterize all quadratic TF distributions and the Wigner-Ville distribution in particular [27]. The kernel function determines the degree of filtering and interference-terms reduction, which is particularly important in coherence analysis since reliable coherence estimates are obtained only if interference terms are completely removed [27]. The kernel therefore determines the temporal and spectral resolution of the TF representations and, as discussed in the next section, the bias of the TF coherence estimates. 


\section{Unbiased time-frequency coherence}

Spectral and TF coherence estimators are known to be biased [29]-[31], as they provide coherence estimates higher than zero for uncorrelated signals. The bias depends on the kernel function and is critically important because it directly affects the coherent and residual spectra through the relation shown in (4). The bias can be described by a function $G$ that maps the theoretical coherence values, $\gamma_{\mathrm{xy}}^{\text {ref }}(t, f)$, to the estimated ones, $\hat{\gamma}_{\mathrm{xy}}(t, f)$, as $\hat{\gamma}_{\mathrm{xy}}(t, f)=G\left(\gamma_{\mathrm{xy}}^{\text {ref }}(t, f)\right)$. Therefore, unbiased coherence estimates, $\tilde{\gamma}_{\mathrm{xy}}(t, f)$, can be estimated as:

$$
\tilde{\gamma}_{\mathrm{xy}}(t, f)=G^{-1}\left(\hat{\gamma}_{\mathrm{xy}}(t, f)\right) \approx \gamma_{\mathrm{xy}}^{\mathrm{ref}}(t, f)
$$

Function $G$ is empirically derived by comparing estimated and theoretical coherence values. A calibration model is used to generate random processes $x(t)$ and $y(t)$ characterized by known theoretical coherence, $\gamma_{\mathrm{xy}}^{\mathrm{ref}}(t, f)$, which is then compared to the estimated coherence, $\hat{\gamma}_{x y}(t, f)$. The calibration model utilized in this paper is the two-input one-output model (see Fig. 1B):

$$
\begin{aligned}
y(t) & =v(t)+n(t)=h(t) * x(t)+n(t) \\
& =\sqrt{a} \cdot \xi_{1}(t)+\sqrt{1-a} \cdot \xi_{2}(t)
\end{aligned}
$$

where (12) is obtained from (11) by choosing $h(t)$ to be equal to the Dirac delta function, $h(t)=\delta(t), x(t)=\sqrt{a} \cdot \xi_{1}(t)$ and $n(t)=\sqrt{1-a} \cdot \xi_{2}(t)$, where $\xi_{1}(t)$ and $\xi_{2}(t)$ are two zero-mean white Gaussian noises with unitary standard deviation, and $a$ is a scaling factor. For this model, the coherence function can be theoretically obtained as [24]:

$$
\gamma_{\mathrm{xy}}^{\mathrm{ref}^{2}}(t, f)=\frac{1}{1+S_{\mathrm{nn}}(t, f) / S_{\mathrm{vv}}(t, f)}=a
$$

In practice, the function $G$ describing the bias associated with a particular TF kernel is empirically estimated as follows:

- Values of $a_{i} \in[0,1]$ with $i=\{1, \ldots, N\}$, are chosen to cover the entire range of possible coherence values.

- For each value of $a_{i}$, the model in (12) is used to generate $j=\{1, \ldots, M\}$ processes $\left\{x_{j}(t), n_{j}(t), y_{j}(t)\right\}$ and $\hat{\gamma}_{\mathrm{xy}, \mathrm{i}, \mathrm{j}}^{2}(t, f)$ is estimated. Then, the mean coherence $\overline{\hat{\gamma}}_{\mathrm{x} y, i}^{2} \in \mathbb{R}$ is obtained by averaging $\hat{\gamma}_{\mathrm{xy}, \mathrm{i}, \mathrm{j}}^{2}(t, f)$ in time, frequency and among the $M$ realizations.

- Function $G$ is derived by comparing $\overline{\hat{\gamma}_{x y, i}^{2}}$ with the theoretical coherence values $a_{i}$.

The inverse function $G^{-1}$ is then used to correct for the bias of any TF coherence function estimated with the same kernel as shown in (10).

\section{Physiological Model and Algorithm Implementation}

Figure 1C shows how the general model described in (1) can be modified to represent QTV as composed of QTVrRRV and QTVuRRV, which are related to supra-ventricular and intrinsic ventricular repolarization dynamics, respectively:

$$
\begin{aligned}
& x_{\mathrm{QT}}(t)=h_{1}(t) * x_{\mathrm{RR}}(t)+h_{2}(t) * x_{\mathrm{VR}}(t)= \\
& =x_{\mathrm{QT}, \mathrm{rRR}}(t)+x_{\mathrm{QT}, \mathrm{rVR}}(t)=x_{\mathrm{QT}, \mathrm{rRR}}(t)+x_{\mathrm{QT}, \mathrm{uRR}}(t)
\end{aligned}
$$

In practice, it is not possible to measure $x_{\mathrm{QT}, \mathrm{rRR}}(t)$ and $x_{\mathrm{QT}, \mathrm{rVR}}(t)$ directly without interfering with normal cardiac physiology. However, information about QTV related to VRD can be obtained by removing from the QTV spectrum the part related to RRV. These are the steps required for its estimation:

- Determine the kernel (8) that provides the most appropriate TF resolution for the analysis and at the same time ensures that $0 \leq \hat{\gamma}_{\mathrm{xy}}(t, f) \leq 1$. Details on how to determine the appropriate kernel's parameters can be found in [30], [32].

- Estimate function $G$ associated with the specific kernel as described in the previous section.

- Estimate the unbiased TF coherence between QTV and RRV, $\tilde{\gamma}_{\mathrm{QT}, \mathrm{RR}}(t, f)$, as in (10).

- Estimate the TF spectrum of QTVuRRV as:

$$
S_{\mathrm{QT}, \mathrm{uRR}}(t, f)=\left(1-\tilde{\gamma}_{\mathrm{QT}, \mathrm{RR}}^{2}(t, f)\right) S_{\mathrm{QT}}(t, f)
$$

where $S_{\mathrm{QT}}(t, f)$ is the TF spectrum of the QTV signal $x_{\mathrm{QTV}}(t)$.

Instantaneous powers and coherence in a given spectral band $\Omega$ are estimated as:

$$
P_{\mathrm{x}}^{\Omega_{\mathrm{P}}}(t)=\int_{\Omega_{\mathrm{P}}} \hat{S}_{\mathrm{xx}}(t, f) d f ; \quad \gamma_{\mathrm{xy}}^{2, \Omega_{\gamma}}(t)=\int_{\Omega_{\gamma}} \hat{\gamma}_{\mathrm{xy}}^{2}(t, f) d f
$$

where $\Omega_{P}$ includes the following spectral bands: $L F \in[0.03-$ $0.15] \mathrm{Hz}, \mathrm{HF} \in[0.15-0.40] \mathrm{Hz}$ and TOT $\in[0.03-0.40] \mathrm{Hz}$, while $\Omega_{\gamma}$ is a time-varying spectral band centered around the frequency of the highest spectral peak of the cross spectrum in LF and HF bands, respectively, and is used to provide a robust measure of the coupling around the instantaneous LF and HF frequencies.

\section{MATERIAL}

\section{A. Simulation study}

The methodology was tested in two simulation studies.

a) Sim1: The model in (12), represented in Fig. 1B, was utilized to simulate the case where TF coherence between output $y(t)$ and input $x(t), \gamma_{x y}^{\text {ref }^{2}}(t)=a(t)$, changes quickly over time, therefore modulating the proportion of the output power related and unrelated to input $x(t)$, denoted as $P_{\mathrm{y}, \mathrm{rx}}(t)$ and $P_{\mathrm{y}, \mathrm{ux}}(t)$, respectively. The temporal evolution of theoretical trends $\gamma_{\mathrm{xy}}^{\mathrm{ref}}(t), P_{\mathrm{y}, \mathrm{rx}}^{\mathrm{ref}}(t)$ and $P_{\mathrm{y}, \mathrm{ux}}^{\mathrm{ref}}(t)$ is shown as a continuous line in Fig. 3. Note that a mixture of abrupt and fast changes over an interval of $5 \mathrm{~min}$ is modeled to assess the methodology in challenging non-stationary conditions.

b) Sim2: The physiological model in (14) (see also Fig. 1C) was modified to simulate challenging conditions where RRV and VRD change quickly as during an exercise stress test. Noise was added to both RRV and QTV. The signal representing RRV, $x_{\mathrm{RR}}(t)$, was a stochastic process generated using a time-varying ARMA scheme that allows to generate ARMA processes with controlled instantaneous central frequencies and spectral amplitudes [33]. Here, each process is composed of an AM LF and an AM-FM HF components as described in Fig. 4A-B. The signal representing intrinsic VRD was modeled as $x_{\mathrm{VR}}(t)=\sigma_{\mathrm{VR}}(t) \xi(t)$, where $\xi(t)$ is a zeromean white Gaussian noise with unitary variance and $\sigma_{\mathrm{VR}}(t)$ is 
an amplitude modulation that determines the dynamic profile of QTVuRRV. The standard deviation of RRV and QTV was adjusted to that of real data (see next section) and set equal to $49.8 \mathrm{~ms}$ and $3.4 \mathrm{~ms}$, respectively. Zero-mean white Gaussian noise with different amplitude was added to both RRV and QTV. Six cases were considered, where the standard deviation of the noise added to RRV was $\sigma_{\mathrm{N}}^{\mathrm{RRV}}=\{0,0.5,1,2,3,4\}$ ms while that added to QTV was twice as big to account for instability in the measurement of the end of the T-wave. The transfer functions were assumed to be scaling factors and QTVuRRV and QTVrRRV contributed equally to total QTV. The instantaneous power of QTVuRRV, $P_{\mathrm{QT}, \mathrm{uRR}}^{\mathrm{TOT}}(t)$, was estimated from the TF representations obtained from 250 realizations of the stochastic model. The relative error and the correlation coefficients between theoretical and estimated trends were calculated within an interval that excludes the first and last $11.7 \mathrm{~s}$ (corresponding to the time resolution). For the sake of comparison, the bias introduced to $P_{\mathrm{QT}, \mathrm{uRR}}^{\mathrm{TOT}}(t)$ by adding noise to QTV and RRV was removed before correlation and error calculations.

\section{B. Autonomic test}

The cardiovascular response to orthostatic challenge was studied in 16 healthy volunteers (aged $29 \pm 3$ years) using a tilt table test. The protocol included early supine (ES) position (4 min), head-up tilt to an angle of $70^{\circ}(5 \mathrm{~min})$, and late supine (LS) position (4 min) as described in other studies [27], [32]. 12-Lead ECG was recorded with a sampling frequency of $1000 \mathrm{~Hz}$. Customized algorithms were used to detect the temporal occurrence of R-waves and T-end. The latter was defined utilizing the tangent method. The QT interval was approximated as the interval from the $\mathrm{R}$-wave to the end of the T-wave in lead V4. Ectopic beats and artifacts were rare. When present, they were removed and the time series were interpolated. RR and QT time series were interpolated at a sampling frequency of $4 \mathrm{~Hz}$, and the RRV and QTV signals, $x_{\mathrm{RR}}(t)$ and $x_{\mathrm{QT}}(t)$, were obtained by high-pass filtering these interpolated series with a cut-off frequency of $0.03 \mathrm{~Hz}$.

\section{Statistical Analysis}

Group results are shown as mean \pm standard deviation for normally distributed data, and as median \pm median absolute deviation for non-normally distributed data. Correlation was measured with the Spearman's correlation coefficient. Wilcoxon signed-rank test was used to assess statistical significance and differences with $P<0.05$ were considered significant.

\section{RESUlts}

\section{A. Coherence bias correction}

The bias of the TF coherence estimator whose kernel provided a time and frequency resolution of $11.7 \mathrm{~s}$ and 0.039 $\mathrm{Hz}$, respectively, is shown in Fig. 2. This was assessed using the scheme described in Section II-C, generating 200 pairs of input/output processes $\{x(t), y(t)\}$ for each theoretical coherence value $a_{i}$. The estimation error was $<5 \%$ for coherence
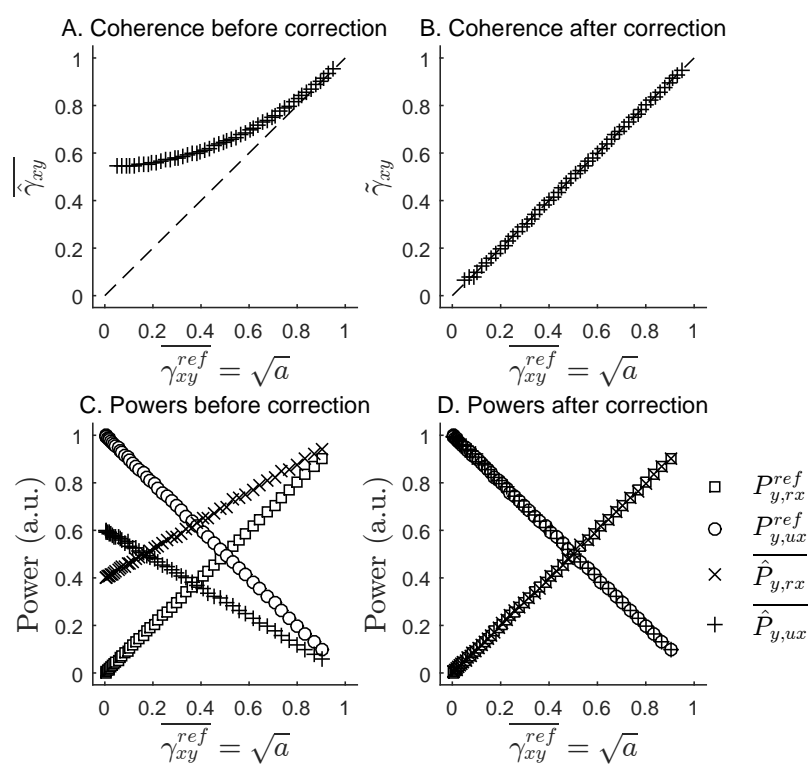

Figure 2. Time-frequency coherence bias correction. Top: Estimated coherence values as a function of theoretical values before (A) and after (B) applying the proposed correction. Bottom: Estimated and theoretical residual and coherent power before (C) and after (D) correction.

values $>0.75$, but it rapidly increased for lower coherence values, with an estimated zero-coherence level $>0.5$. The estimation error in the coherence function introduced a bias in the power of the residual and coherent spectra as well (Fig. 2C). The algorithm proposed for correcting the TF coherence bias provided accurate coherence and power estimates with an almost perfect agreement between theoretical and estimated values (Fig. 2B,D).

\section{B. Simulation Studies}

Figure 3 shows the results of Sim1, which demonstrate that the method for correcting for the TF coherence bias provides accurate estimates. Mean trends of $\gamma_{\mathrm{xy}}^{\mathrm{TOT}}(t), P_{\mathrm{y}, \mathrm{rx}}^{\mathrm{TOT}}(t)$ and $P_{\mathrm{y}, \mathrm{ux}}^{\mathrm{TOT}}(t)$, calculated from mean $\mathrm{TF}$ representations obtained from 100 realizations of the model in (12), are shown along with their corresponding theoretical values. Before correcting for the bias (panels on the left), estimated trends correlated well with the theoretical patterns (correlation coefficients $>0.96$ ), but the relative estimation error was high, being equal to $24 \% \pm 25 \%$ for $\gamma_{\mathrm{xy}}^{\mathrm{TOT}}(t), 103 \% \pm 94 \%$ for $P_{\mathrm{y}, \mathrm{rx}}^{\mathrm{TOT}}(t)$ and $-36 \% \pm 10 \%$ for $P_{\mathrm{y} \text {,ux }}^{\mathrm{TOT}}(t)$ (mean \pm standard deviation). After correcting for the bias (panels on the right), the correlation coefficients only marginally improved to $>0.97$ for all the three indices, while the estimation error dramatically decreased to $-2 \% \pm 11 \%$ for $\gamma_{x y}^{\mathrm{TOT}}(t), 0 \% \pm 23 \%$ for $P_{\mathrm{y}, \mathrm{rx}}^{\mathrm{TOT}}(t)$ and $5 \% \pm 18 \%$ for $P_{\mathrm{y}, \mathrm{ux}}^{\mathrm{TOT}}(t)$. This estimation error was mainly due to a minor delay in tracking sudden changes (see Fig. 3). Figure 4 shows the TF structure of the RRV signals utilized in Sim2 (panels A-B), a representative example of simulated RRV and QTV signals (panel C), theoretical and estimated trends of QTVuRRV in the absence of noise (panel D) and when noise was added to both RRV and QTV (panel E). Numerical results are shown in Table I. In the absence of noise, 
Table I

RESUltS OF SIM2: $\sigma$ : AMPLITUdE OF WHITE-GAUSSIAN NOISE ADDED TO RRV AND QTV AND ASSOCIATED WITH CORRESPONDING SNR. ACCURACY IN TRACKING $P_{\mathrm{QT}, \mathrm{uRR}}^{\mathrm{TOT}}(t)$ IS ASSESSED BY: CORRELATION COEFFICIENT $(r)$, MEAN $\left(E_{\mathrm{M}}\right)$, STANDARD DEVIATION $\left(E_{\mathrm{SD}}\right)$, MEDIAN $\left(E_{\mathrm{MED}}\right)$ AND MEDIAN ABSOLUTE DEVIATION ( $E_{\text {MAD }}$ ) OF THE DIFFERENCE BETWEEN ESTIMATED AND THEORETICAL TRENDS (EST-VS-THEO) AND BETWEEN ESTIMATED TRENDS AND TRENDS DERIVED FROM THE TF SPECTRUM OF $x_{\mathrm{QT}, \mathrm{uRR}}(t)$ (EST-VS-OPTIMAL).

\begin{tabular}{|c|c|c|c|c|c|c|c|c|c|c|c|c|c|}
\hline \multicolumn{4}{|c|}{ Param } & \multicolumn{5}{|c|}{ Est-vs-Theo } & \multicolumn{5}{|c|}{ Est-vs-Optimal } \\
\hline $\begin{array}{c}\sigma_{\mathrm{N}}^{\mathrm{RRV}} \\
(\mathrm{ms})\end{array}$ & $\begin{array}{l}\sigma_{\mathrm{N}}^{\mathrm{QTV}} \\
(\mathrm{ms})\end{array}$ & $\begin{array}{c}\mathrm{SNR}_{\mathrm{RRV}} \\
(\mathrm{dB})\end{array}$ & $\begin{array}{c}\mathrm{SNR}_{\mathrm{QTV}} \\
(\mathrm{dB})\end{array}$ & $\begin{array}{c}r \\
(\mathrm{nu})\end{array}$ & $\begin{array}{l}E_{\mathrm{M}} \\
(\%)\end{array}$ & $\begin{array}{c}E_{\mathrm{SD}} \\
(\%)\end{array}$ & $\begin{array}{c}E_{\mathrm{MED}} \\
(\%)\end{array}$ & $\begin{array}{c}E_{\mathrm{MAD}} \\
(\%)\end{array}$ & $\begin{array}{c}r \\
(\mathrm{nu})\end{array}$ & $\begin{array}{l}E_{\mathrm{M}} \\
(\%)\end{array}$ & $\begin{array}{c}E_{\mathrm{SD}} \\
(\%)\end{array}$ & $\begin{array}{c}E_{\mathrm{MED}} \\
(\%)\end{array}$ & $\begin{array}{c}E_{\mathrm{MAD}} \\
(\%)\end{array}$ \\
\hline 0.0 & 0.0 & Inf & Inf & 0.99 & 10.57 & 35.65 & -0.74 & 5.08 & 1.00 & -1.89 & 6.19 & 0.56 & 1.46 \\
\hline 0.5 & 1.0 & 40.0 & 7.6 & 0.99 & 12.64 & 36.88 & 0.72 & 4.64 & 1.00 & -0.85 & 3.60 & 0.00 & 1.72 \\
\hline 1.0 & 2.0 & 33.9 & 1.7 & 0.98 & 11.51 & 36.16 & 0.27 & 5.22 & 1.00 & -2.87 & 4.74 & -0.89 & 2.50 \\
\hline 2.0 & 4.0 & 27.9 & -4.4 & 0.95 & 12.79 & 38.71 & -1.40 & 12.75 & 0.97 & 0.07 & 14.63 & -3.10 & 7.23 \\
\hline 3.0 & 6.0 & 24.4 & -7.9 & 0.94 & 19.73 & 56.11 & 2.11 & 16.99 & 0.96 & 4.92 & 31.20 & 2.07 & 13.33 \\
\hline 4.0 & 8.0 & 21.9 & -10.4 & 0.92 & 12.78 & 77.37 & 2.03 & 16.16 & 0.94 & -1.26 & 49.99 & 0.70 & 13.48 \\
\hline
\end{tabular}
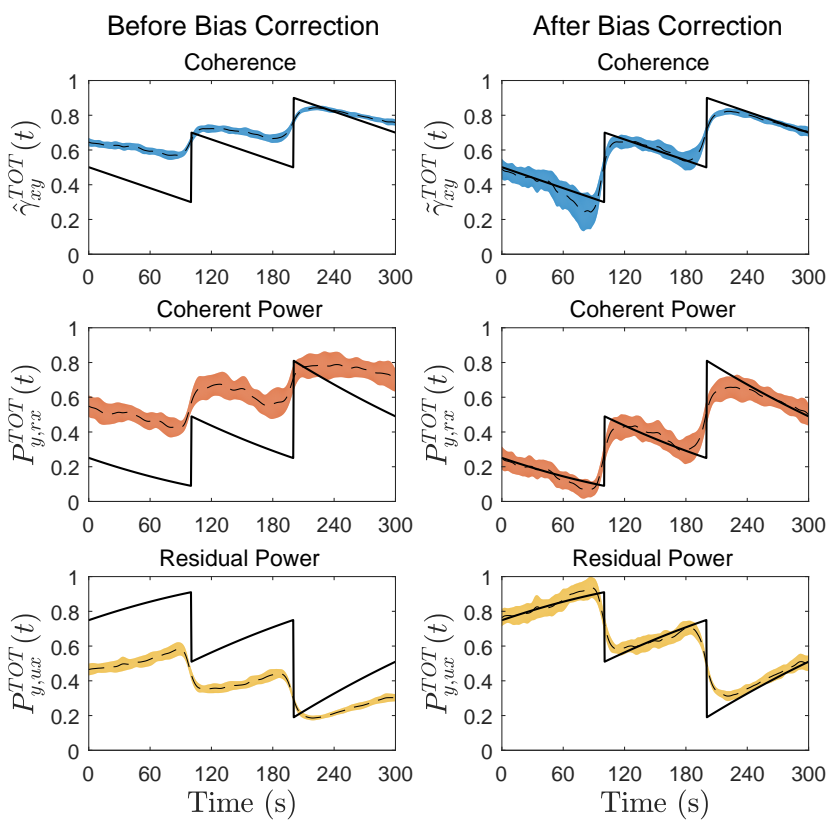

Figure 3. Results of Sim1. Coherence, residual and coherent power before (left) and after (right) correcting for the TF bias using the proposed methodology. Solid and dashed lines represent theoretical and estimated mean values, respectively. Shadowed areas represent standard deviation intervals.

the correlation coefficient between $P_{\mathrm{QT}, \mathrm{uRR}}^{\mathrm{TOT}}(t)$ estimated using the proposed methodology (continuous red line) and the theoretical trend obtained from its analytical formulation (dotted line) was equal to 0.99 , while the relative error was equal to $10.57 \% \pm 35.65 \%$ (mean \pm standard deviation). The error was almost entirely due to a delay in the tracking of the abrupt changes of the theoretical trend of $P_{\mathrm{QT}, \mathrm{uRR}}^{\mathrm{TOT}}(t)$, which is due to the TF smoothing necessary to estimate a time-varying spectrum using a Cohen's class distribution [27]. In fact, the error decreased to $-1.89 \% \pm 6.19 \%$ and the correlation coefficient increased to 1.00 when comparing $P_{\mathrm{QT}, \mathrm{uRR}}^{\mathrm{TOT}}(t)$ estimated using the proposed methodology with $P_{\mathrm{QT}, \mathrm{uRR}}^{\mathrm{T} T \mathrm{~T}}(t)$ directly estimated from the TF spectrum of $x_{\mathrm{QT}, \mathrm{uRR}}(t)$, which represents the optimal trend (bold dashed line). When adding noise, the SNR was more than $32 \mathrm{~dB}$ lower in QTV than in RRV, due to the imbalance between the power content of the two signals and the higher instability in the detection of
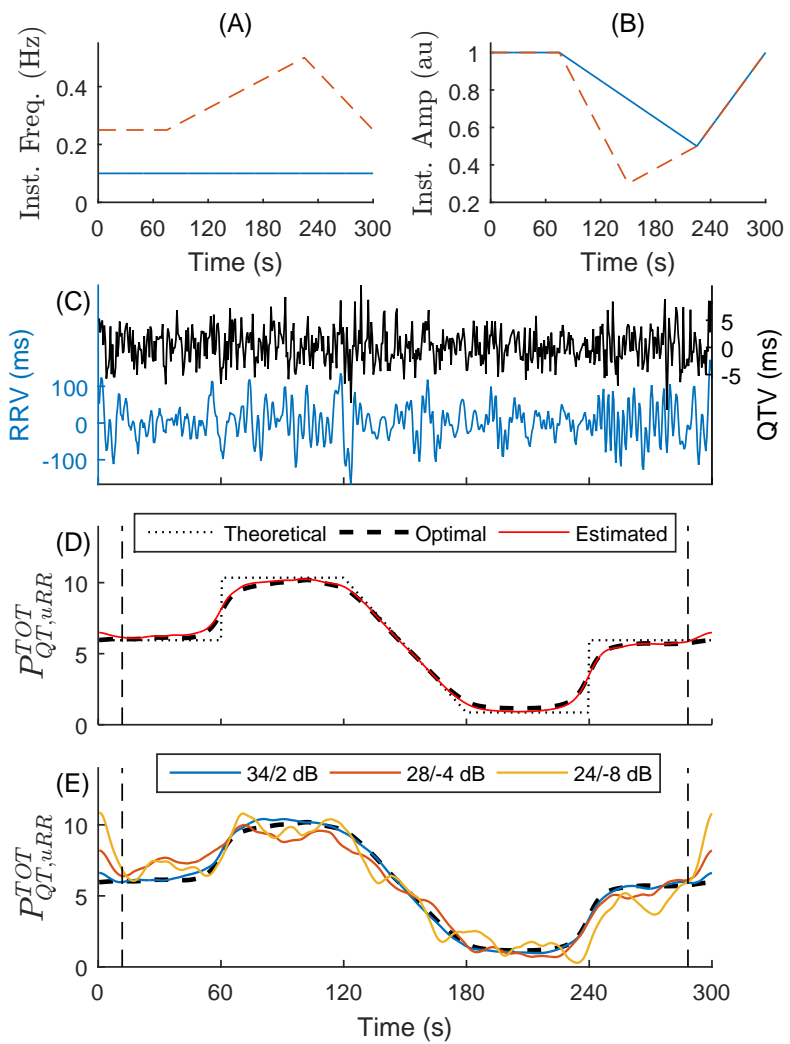

Figure 4. Results of Sim2. A-B: Instantaneous central frequencies and amplitudes of ARMA processes mimicking RRV during exercise stress test. C: Examples of stochastic processes representing RRV and QTV. D: Temporal evolution of QTVuRRV in the absence of noise, with theoretical values obtained from the analytical formulation of VRD (dotted line), theoretical values estimated from the TF spectrum of $x_{\mathrm{QT}, \mathrm{uRR}}(t)$ (bold dashed line) and QTVuRRV estimated from the mean residual TF spectrum (continuous red line). E: Estimation of QTVuRRV in the presence of noise, with SNR ratio for RRV/QTV given in the legend. Vertical lines enclose the interval considered for correlation and error calculations.

the T-end with respect to the R-peak (Table I). Nevertheless, the correlation between the estimated and theoretical trends remained $\geq 0.92$ for all cases, while the standard deviation of the differences between the estimated and the optimal trends was $\leq 15 \%$ for QTV with SNR $\geq-4 \mathrm{~dB}$ (Table I), but increased for lower SNR. 


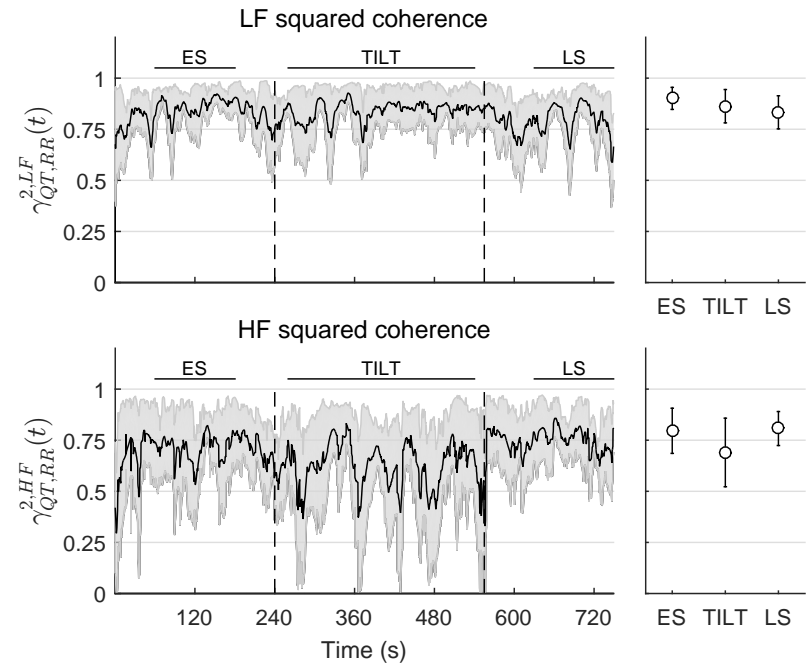

Figure 5. Results of the tilt table test. Temporal evolution of squared spectral coherence. Panels on the left: Continuous line and shaded areas represent median trends and median absolute deviation among patients, respectively. Panels on the right: Circles and bars represent median and median absolute deviation of the mean instantaneous squared coherence calculated within the intervals represented by horizontal lines on the left hand side panels.

\section{Autonomic Test}

During the tilt table test, the correlation coefficient between QTV and RRV was equal to $0.68 \pm 0.14$, and did not change significantly between supine and head-up position. This suggests that although RRV accounted for the largest part of QTV, a substantial part of repolarization dynamics were not linearly related to heart rate variability.

The median trend of the squared spectral coherence was $\gamma_{\mathrm{QT}, \mathrm{RR}}^{2}(t)>0.50$ in both LF and HF during the entire test (Fig. 5), confirming the predominance of QTVrRRV with respect to QTVuRRV. However, high inter-subject variability was observed. During tilt, squared spectral coherence slightly decreased with respect to the early supine position in both LF $(0.90 \pm 0.05$ vs $0.86 \pm 0.08, P=0.21)$ and $\mathrm{HF}(0.80 \pm 0.11$ vs $0.69 \pm 0.17, P=0.20$ ), yet without reaching statistical significance. Squared spectral coherence tended to be higher in LF than in HF during both supine position $(P=0.05)$ and tilt $(P=0.02)$.

$P_{\mathrm{QT}, \mathrm{uRR}}(t)$ and $P_{\mathrm{QT}, \mathrm{rRR}}(t)$ showed a similar trend in all spectral bands. Both coherent and residual power increased very quickly after tilt and decreased more slowly towards baseline values once the supine position was restored (Fig. 6). Changes were more pronounced in $P_{\mathrm{QT}, \mathrm{uRR}}(t)$ than in $P_{\mathrm{QT}, \mathrm{rRR}}(t)$. Analysis of mean QTV power during supine position and tilt (see intervals shown in Fig. 6) revealed that during tilt QTV and QTVuRRV significantly increased with respect to early supine values in all spectral bands $(P<0.01)$, while QTVrRRV did not increase significantly in HF (Fig. 7A-C). Interestingly, during tilt the relative increase in QTVuRRV was greater than the relative increase in QTVrRRV or in global QTV in all spectral bands $(P \approx 0.05$, see Fig. 7DF), with a ratio between tilt versus early supine values equal to $2.19 \pm 1.2$ for QTVuRRV, $1.95 \pm 0.7$ for QTV, and $1.45 \pm 0.7$ for QTVrRRV (median \pm median absolute deviation).
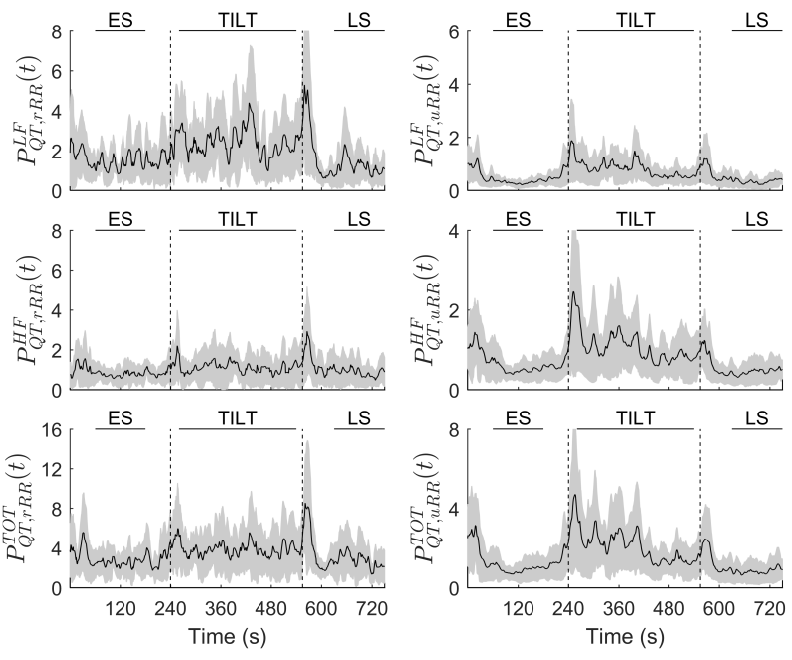

Figure 6. Trends during tilt table test. Instantaneous powers of QTV related, $P_{\mathrm{QT}, \mathrm{rRR}}(t)$, and unrelated, $P_{\mathrm{QT}, \mathrm{uRR}}(t)$, to RRV in LF, HF and TOT spectral bands are shown on the left and right panels, respectively. Solid lines represent median values, and shaded areas represent median \pm median absolute deviation. Note that the range of the vertical axes is not the same for all panels.



Figure 7. Global results for the tilt table test. Top: Median \pm median absolute deviation of mean QTV, $P_{\mathrm{QT}}(t)$, QTV related, $P_{\mathrm{QT}, \mathrm{rRR}}(t)$, and unrelated, $P_{\mathrm{QT}, \mathrm{uRR}}(t)$, to RRV during early supine (ES), tilt (TILT) and late supine (LS) phases (see horizontal lines in Fig. 6). *: Statistically significant differences with respect to ES values. Bottom: Changes of $P_{\mathrm{QT}}(t)$, $P_{\mathrm{QT}, \mathrm{rRR}}(t)$ and $P_{\mathrm{QT}, \mathrm{uRR}}(t)$ with respect to ES values. P-values are shown in round brackets.

\section{Discussion}

This paper presents a novel methodology to separate the non-stationary spectrum of QTV into two parts, one locally linearly coupled and another locally linearly uncoupled to RRV. The methodology is based on Cohen's class TF distributions and incorporates a novel algorithm to correct for the TF coherence bias. The main results are: Two simulation studies demonstrated that the methodology is robust and accurate, and is able to track changes in QTV unrelated to RRV with only a minor delay due to the TF filtering necessary to estimate the non-stationary spectra. Correlation coefficients between estimated and theoretical patterns were $\geq 0.92$ even 
for extremely noisy conditions. The analysis of ECG data recorded during an autonomic test showed that the increase in QTV during tilt was driven by both QTV related and unrelated to $\mathrm{RRV}$, with the latter showing the greatest relative increase during tilt.

\section{A. Methodological considerations}

This is the first study to present a comprehensive description of this methodology as a TF extension of classical multipleinput single-output theory [24] and it includes a novel algorithm to correct for the TF coherence bias. This extends previous work where residual TF spectra and coherence were applied to study cardio-respiratory and cardiovascular interactions [28], [34], [35]. The bias correction proposed in this paper is crucial to accurately estimate residual and coherent spectra, because these are obtained by multiplying the output spectrum by the squared TF coherence function. Spectral coherence estimators are known to be biased [29]-[31]. This has led to propose methodologies based on surrogate data analysis to estimate a threshold for determining significant levels of coherence [27], [29], [36]. However, to the extent of our knowledge, this is the first paper to present a scheme to estimate unbiased TF coherence functions. The proposed scheme assumes that the bias depends on the estimator's parameters and is independent of the TF properties of the signals. The results of the simulation studies support this assumption. Previous studies on the interaction between QTV and RRV have mainly utilized time-invariant autoregressive models [19], [21], [22], [37] where RRV contributes to QTV, which in turn does not contribute to RRV. A recent study implementing a network physiology approach to assess strength and directionality of sino-atrial and ventricular interactions found a strong causal link from RRV to QTV and a weak link in the reverse direction [38]. This is in agreement with our simple open-loop model, where QTV is the sum of intrinsic repolarization and heart period dynamics.

It is worth noting that non-physiological variability due to inaccurate measurement of ECG intervals would still be assigned to one of these two components.

Model-free TF estimators have the advantage of not relying upon the ability of an underlying model to capture the data dynamics, but they mainly provide information related to time-varying spectral power and coherence, while spectral model-based approach can be used to infer directionality or causality [36], [38]-[41]. A comparison with time-varying autoregressive methods is beyond the scope of this paper, but previous work has suggested that TF distributions may be more accurate in tracking highly non-stationary dynamics [42], [43]. Finally, the methodology presented in this paper can be applied to any dynamic system that can be described by a two-input one-output model with uncorrelated inputs.

\section{B. Physiological Considerations and Clinical perspective}

The model presented in this paper decomposes QTV into two components, one related and another unrelated to RRV. Variations in QTV are therefore determined by the changes in these two components, whose magnitude and direction depend on changes in both the magnitude of QTV-RRV coherence and global QTV. The component unrelated to RRV is assumed to be due to intrinsic ventricular repolarization dynamics uncorrelated to RRV. Although heart rate and ventricular repolarization stem from different anatomical structures that may receive independent autonomic stimulation, common drivers cannot be excluded since respiratory and LF oscillations are common in RRV and have also been observed in the human ventricular repolarization [44], [45]. Although both RRV and QTV are known to be affected by sympathetic modulation [1], the degree of correlation between sympathetic outflow directed to the sinoatrial node and the ventricles is unknown. Interestingly, recent studies have shown that sympathetic activity can induce LF oscillations in ventricular repolarization, part of which are unrelated to RRV [46], with strong predictive value in post MI patients [46], [47].

Enhanced intrinsic repolarization variability is thought to be driven by imbalanced ion channel dynamics at the level of the ventricular myocyte, including calcium [15] and potassium [16] dynamics, which are modulated by sympathetic nervous activity. A methodology that provides non-invasive assessment of intrinsic VRD by measuring QTV unrelated to heart rate variability may find applications in arrhythmic risk stratification [1], [9], [13], [48] as well as in the noninvasive assessment of sympathetic ventricular activity [19], [49]. For instance, correlations between QTV and a direct measure of sympathetic activity were observed in patients with hypertension [50], while patients with heart failure and spontaneous ventricular tachycardia have higher QTV than control [51].

Passive tilt is an established model to study the autonomic modulation of cardiac activity, since it induces a shift of the sympathovagal balance toward sympathetic predominance [49], [52]. Our results are in partial agreement with a previous study where healthy volunteers underwent a graded headup tilt test with different tilt table inclinations [49]. In [49], spectral coherence between QTV and RRV was higher in LF than $\mathrm{HF}$ and only decreased in HF for tilting angle between 60 to 90 . A longer duration of the head-up tilt phase (10 min in [49]) may partially justify the fact that in the present study the coherence showed a trend toward lower values during tilt without reaching statistical significance.

Although the effect of adrenergic stimulation on ventricular repolarization is well established [17], [53], its assessment in patients is challenging, mainly due to the difficulty of directly measuring sympathetic outflow to the ventricle. In a recent study, muscle sympathetic nerve activity measured in the peroneal nerve, one of the few established direct measures of sympathetic activity, did not contribute significantly to QTV during rest or head-up tilt at 40 degrees [22]. Own to its prominent role in sudden cardiac death [46], [54], the interaction between sympathetic modulation and repolarization dynamics deserves further investigation.

\section{Limitation and further studies}

The proposed methodology does not account for any contribution of respiration not mediated by respiratory sinus arrhythmia, i.e. RRV in synchrony with respiration. In our approach, 
a three-input/one-output model including respiration cannot be implemented due to the high correlation between respiration and RRV, which violates the assumption of uncorrelation between the inputs. To assess to which extent this limitation may affect the results, further analysis was conducted including the respiratory signal, which was simultaneously recorded during the test (see Supplementary Document 1 for a detailed discussion of the results). In brief, the coherence between RRV and respiration was high and explained most of the respiratory sinus arrhythmia variability (Supplementary Fig. $\mathrm{S} 1$ ). In the respiratory spectral band, the coherence between QTV and RRV and between QTV and respiration were similar and highly correlated (Supplementary Fig. S2 and S3A). Consequently, QTVuRRV and QTV unrelated to respiration were also similar and highly correlated (Supplementary Fig. S2B and S3B). This suggests that QTV related to respiration but not accounted for by respiratory sinus arrhythmia was small as compared to the portion accounted for by respiratory sinus arrhythmia. Therefore, although the proposed methodology is not able to directly remove from QTV respiratory components unrelated to RRV, this limitation is expected to have little effect on the results. Multi-lead strategies for ECG waves delineation can reduce this source of variability [55], but in applications where this component needs to be directly accounted for or entirely removed, other techniques may be preferred [19].

The proposed methodology is linear and therefore unable to capture and remove QTV dynamics non-linearly related to RRV, which are known to be present [21]. Further studies should assess to which extent this limitation affects the results and compare or integrate the proposed methodology with those able to capture these dynamics [1], [41], [56].

In this study, the same signal-independent kernel has been used to estimate all TF representations. Future studies may investigate whether better results could be achieved using signal-dependent approaches [30] where the TF resolution could be adjusted to the high inter-subject variability of repolarization dynamics, including rate-adaptation and autonomic modulation.

\section{CONCLUSION}

This novel methodology provides accurate dynamic assessment of QTV unrelated to RRV, which may provide useful information for assessing intrinsic ventricular repolarization variability, an important marker of cardiac instability associated with sympathetic ventricular modulation and sudden cardiac death.

\section{REFERENCES}

[1] M. Baumert, A. Porta, M. A. Vos, M. Malik, J.-P. Couderc, P. Laguna, G. Piccirillo, G. L. Smith, L. G. Tereshchenko, and P. G. Volders, "QT interval variability in body surface ECG: measurement, physiological basis, and clinical value: position statement and consensus guidance endorsed by the european heart rhythm association jointly with the esc working group on cardiac cellular electrop," Europace, pp. 925-944, 2016.

[2] X. Zhou, A. Bueno-Orovio, M. Orini, B. Hanson, M. Hayward, P. Taggart, P. D. Lambiase, K. Burrage, and B. Rodriguez, "In vivo and in silico investigation into mechanisms of frequency dependence of repolarization alternans in human ventricular cardiomyocytesnovelty and significance," Circ. Res., vol. 118, no. 2, pp. 266-278, 2016.
[3] M. Orini, B. Hanson, V. Monasterio, J. Martinez, M. Hayward, P. Taggart, and P. Lambiase, "Comparative evaluation of methodologies for t-wave alternans mapping in electrograms," IEEE Trans. Biomed. Eng., vol. 61, no. 2, pp. 308-316, 2014.

[4] J.-M. Cao, Z. Qu, Y.-H. Kim, T.-J. Wu, A. Garfinkel, J. Weiss, H. Karagueuzian, and P.-S. Chen, "Spatiotemporal heterogeneity in the induction of ventricular fibrillation by rapid pacing: Importance of cardiac restitution properties," Circ. Res., vol. 84, no. 11, pp. 1318-1331, 1999.

[5] J. Ramírez, M. Orini, A. Mincholé, V. Monasterio, I. Cygankiewicz, A. Bayés de Luna, J. P. Martínez, E. Pueyo, and P. Laguna, "T?Wave Morphology Restitution Predicts Sudden Cardiac Death in Patients With Chronic Heart Failure," Journal of the American Heart Association, vol. 6, no. 5, p. e005310, 2017.

[6] E. Pueyo, Z. Husti, T. Hornyik, I. Baczkó, P. Laguna, A. Varró, and B. Rodríguez, "Mechanisms of ventricular rate adaptation as a predictor of arrhythmic risk." Am J Physiol Heart Circ Physiol, vol. 298, no. 5, pp. H1577-87, 2010.

[7] E. Pueyo, P. Smetana, P. Caminal, A. DeLuna, M. Malik, and P. Laguna, "Characterization of QT Interval Adaptation to RR Interval Changes and Its Use as a Risk-Stratifier of Arrhythmic Mortality in AmiodaroneTreated Survivors of Acute Myocardial Infarction," IEEE Trans. Biomed. Eng., vol. 51, no. 9, pp. 1511-1520, 2004.

[8] P. Laguna, J. P. Martinez, and E. Pueyo, "Techniques for Ventricular Repolarization Instability Assessment From the ECG," Proceedings of the IEEE, vol. 104, no. 2, pp. 392-415, 2016.

[9] R. Varkevisser, S. C. Wijers, M. A. van der Heyden, J. D. Beekman, M. Meine, and M. A. Vos, "Beat-to-beat variability of repolarization as a new biomarker for proarrhythmia in vivo," Heart Rhythm, vol. 9, no. 10, pp. 1718-1726, 2012.

[10] M. N. Niemeijer, M. E. van den Berg, M. Eijgelsheim, G. van Herpen, B. H. Stricker, J. A. Kors, and P. R. Rijnbeek, "Short-term QT variability markers for the prediction of ventricular arrhythmias and sudden cardiac death: a systematic review," Heart, vol. 100, no. 23, pp. 1831-1836, 2014.

[11] L. G. Tereshchenko, B. J. Fetics, P. P. Domitrovich, B. D. Lindsay, and R. D. Berger, "Prediction of ventricular tachyarrhythmias by intracardiac repolarization variability analysis," Circ Arrhythm Electrophysiol, vol. 2, no. 3, pp. 276-284, 2009.

[12] G. Piccirillo, F. Moscucci, G. D’Alessandro, M. Pascucci, P. Rossi, S. Han, L. Chen, S.-F. Lin, P.-S. Chen, and D. Magr, "Myocardial repolarization dispersion and autonomic nerve activity in a canine experimental acute myocardial infarction model," vol. 11, no. 1, pp. 110-118, 2014.

[13] M. B. Thomsen, P. G. A. Volders, J. D. M. Beekman, J. Matz, and M. A. Vos, "Beat-to-Beat Variability of Repolarization Determines Proarrhythmic Outcome in Dogs Susceptible to Drug-Induced Torsades de Pointes," J Am Coll Cardiol, vol. 48, no. 6, pp. 1268-1276, 2006.

[14] M. Orini, P. Taggart, N. Srinivasan, M. Hayward, and P. D. Lambiase, "Interactions between activation and repolarization restitution properties in the intact human heart: In-vivo whole-heart data and mathematical description," PLOS ONE, vol. 11, no. 9, p. e0161765, 2016.

[15] D. M. Johnson, J. Heijman, E. F. Bode, D. J. Greensmith, H. van der Linde, N. Abi-Gerges, D. A. Eisner, A. W. Trafford, and P. G. A. Volders, "Diastolic spontaneous calcium release from the sarcoplasmic reticulum increases beat-to-beat variability of repolarization in canine ventricular myocytes after $\beta$-adrenergic stimulation." Circ. Res., vol. 112, no. 2, pp. 246-256, 2013.

[16] E. Pueyo, A. Corrias, L. Virág, N. Jost, T. Szél, A. Varró, N. Szentandrássy, P. P. Nánási, K. Burrage, and B. Rodríguez, “A multiscale investigation of repolarization variability and its role in cardiac arrhythmogenesis," Biophysical Journal, vol. 101, no. 12, pp. 2892-2902, 2011.

[17] E. Pueyo, M. Orini, J. F. Rodriguez, and P. Taggart, "Interactive effect of beta-adrenergic stimulation and mechanical stretch on low-frequency oscillations of ventricular action potential duration in humans," $\mathrm{J} \mathrm{Mol}$ Cell Cardiol, vol. 97, pp. 93 - 105, 2016.

[18] J. Heijman, A. Zaza, D. M. Johnson, Y. Rudy, R. L. M. Peeters, P. G. A. Volders, and R. L. Westra, "Determinants of beat-to-beat variability of repolarization duration in the canine ventricular myocyte: a computational analysis." PLoS computational biology, vol. 9, no. 8, p. e1003202, 2013.

[19] A. Porta, E. Tobaldini, T. Gnecchi-Ruscone, and N. Montano, "RT variability unrelated to heart period and respiration progressively increases during graded head-up tilt," Am J Physiol Heart Circ Physiol, vol. 298, no. 5, pp. H1406-H1414, 2010. 
[20] M. Baumert, B. Czippelova, A. Porta, and M. Javorka, "Decoupling of QT interval variability from heart rate variability with ageing," Physiol. Meas., vol. 34, no. 11, pp. 1435-1448, 2013.

[21] R. Almeida, S. Gouveia, A. Rocha, E. Pueyo, J. Martinez, and P. Laguna, "QT variability and HRV interactions in ECG: Quantification and reliability," IEEE Trans. Biomed. Eng., vol. 53, no. 7, pp. 1317-1329, 2006.

[22] F. El-Hamad, E. Lambert, D. Abbott, and M. Baumert, "Relation between QT interval variability and muscle sympathetic nerve activity in normal subjects," Am J Physiol Heart Circ Physiol, vol. 309, no. 7, pp. H1218-H1224, 2015.

[23] G. Valenza, M. Orini, L. Citi, A. Mincholé, E. Pueyo, P. Laguna, and R. Barbieri, "Assessing real-time RR-QT frequency-domain measures of coupling and causality through inhomogeneous point-process bivariate models." Conf Proc IEEE Eng Med Biol Soc., vol. 2014, pp. 6475-6478, 2014.

[24] J. S. Bendat and A. G. Piersol, "Multiple-input/output relationships," in Random Data. John Wiley \& Sons, Inc., 2012, pp. 201-247.

[25] G. Baselli, A. Porta, and S. Cerutti, "Spectral decomposition in multichannel recordings based on multivariate parametric identification," IEEE Trans. Biomed. Eng., vol. 44, no. 11, pp. 1092-1101, 1997.

[26] G. C. Carter, "Coherence and time delay estimation," Proceedings of the IEEE, vol. 75, no. 2, pp. 236-255, 1987.

[27] M. Orini, R. Bailon, L. T. Mainardi, P. Laguna, and P. Flandrin, "Characterization of dynamic interactions between cardiovascular signals by time-frequency coherence." IEEE Trans. Biomed. Eng., vol. 59, no. 3, pp. 663-673, 2012.

[28] M. Orini, R. Bailon, P. Laguna, L. Mainardi, and R. Barbieri, "A multivariate time-frequency method to characterize the influence of respiration over heart period and arterial pressure," EURASIP Journal on Advances in Signal Processing, vol. 2012, no. 1, p. 214, 2012.

[29] L. Faes, G. D. Pinna, A. Porta, R. Maestri, and G. Nollo, "Surrogate data analysis for assessing the significance of the coherence function," IEEE Trans. Biomed. Eng., vol. 51, no. 7, pp. 1156-1166, 2004.

[30] M. Orini, R. Bailón, L. Mainardi, A. Mincholé, and P. Laguna, "Continuous quantification of spectral coherence using quadratic time-frequency distributions: error analysis and application," Internat. Conf. Computers in Cardiology, pp. 681-684, 2009.

[31] K. Keissar, L. R. Davrath, and S. Akselrod, "Coherence analysis between respiration and heart rate variability using continuous wavelet transform," Philos Trans A Math Phys Eng Sci, vol. 367, no. 1892, pp. 1393-1406, 2009.

[32] M. Orini, P. Laguna, L. T. Mainardi, and R. Bailón, "Assessment of the dynamic interactions between heart rate and arterial pressure by the cross time-frequency analysis." Physiol. Meas., vol. 33, no. 3, pp. 315-331, 2012.

[33] M. Orini, R. Bailón, L. Mainardi, and P. Laguna, "Synthesis of HRV signals characterized by predetermined time-frequency structure by means of time-varying ARMA models," Biomed Signal Process Control, vol. 7, no. 2, pp. $141-150,2012$.

[34] M. Orini, P. Taggart, and P. D. Lambiase, "A multivariate time-frequency approach for tracking QT variability changes unrelated to heart rate variability," Conf Proc IEEE Eng Med Biol Soc., pp. 924-927, 2016.

[35] D. Widjaja, M. Orini, E. Vlemincx, and S. Van Huffel, "Cardiorespiratory dynamic response to mental stress: A multivariate time-frequency analysis," Comput Math Methods Med, vol. 2013, p. 12, 2013.

[36] L. Faes, A. Porta, and G. Nollo, "Testing frequency-domain causality in multivariate time series," IEEE Trans. Biomed. Eng., vol. 57, no. 8, pp. 1897-1906, 2010.

[37] A. Porta, G. Baselli, E. Caiani, A. Malliani, F. Lombardi, and S. Cerutti, "Quantifying electrocardiogram RT-RR variability interactions," Med. Biol. Eng. Comput., vol. 36, no. 1, pp. 27-34, 1998.

[38] A. Porta, V. Bari, B. De Maria, and M. Baumert, "A network physiology approach to the assessment of the link between sinoatrial and ventricular cardiac controls," Physiological Measurement, vol. 38, no. 7, pp. 1472 1489, jun 2017.

[39] L. Faes, G. Nollo, and A. Porta, "Information domain approach to the investigation of cardio-vascular, cardio-pulmonary and vasculopulmonary causal couplings," Frontiers in Physiology, vol. 2, no. 0, 2011.

[40] L. Faes, S. Erla, A. Porta, and G. Nollo, "A framework for assessing frequency domain causality in physiological time series with instantaneous effects," Philos Trans A Math Phys Eng Sci, vol. 371, no. 1997, 2013.

[41] A. Porta and L. Faes, "Wiener-granger causality in network physiology with applications to cardiovascular control and neuroscience," Proceedings of the IEEE, vol. 104, no. 2, pp. 282-309, 2016.
[42] S. Pola, A. Macerata, M. Emdin, and C. Marchesi, "Estimation of the power spectral density in nonstationary cardiovascular time series: assessing the role of the time-frequency representations (TFR)," IEEE Trans. Biomed. Eng., vol. 43, no. 1, p. 46, 1996.

[43] M. Orini, R. Bailon, P. Laguna, and L. T. Mainardi, "Modeling and estimation of time-varying heart rate variability during stress test by parametric and non parametric analysis," in Proc. Computers in Cardiology, 2007, pp. 29-32.

[44] B. Hanson, N. Child, S. Van Duijvenboden, M. Orini, Z. Chen, R. Coronel, C. A. Rinaldi, J. S. Gill, J. S. Gill, and P. Taggart, "Oscillatory behavior of ventricular action potential duration in heart failure patients at respiratory rate and low frequency." Front Physiol, vol. 5, p. 414, 2014.

[45] S. van Duijvenboden, B. Hanson, N. Child, M. Orini, C. A. Rinaldi, J. S. Gill, and P. Taggart, "Effect of autonomic blocking agents on the respiratory-related oscillations of ventricular action potential duration in humans." Am J Physiol Heart Circ Physiol, vol. 309, no. 12, pp. H2108-H2117, 2015.

[46] K. D. Rizas, T. Nieminen, P. Barthel, C. S. Zürn, M. Kähönen, J. Viik, T. Lehtimäki, K. Nikus, C. Eick, T. O. Greiner, H. P. Wendel, P. Seizer, J. Schreieck, M. Gawaz, G. Schmidt, and A. Bauer, "Sympathetic activity-associated periodic repolarization dynamics predict mortality following myocardial infarction," J. Clin. Invest., vol. 124, no. 4, pp. 1770-1780, 2014

[47] K. D. Rizas, S. McNitt, W. Hamm, S. Massberg, S. Kääb, W. Zareba, J.P. Couderc, and A. Bauer, "Prediction of sudden and non-sudden cardiac death in post-infarction patients with reduced left ventricular ejection fraction by periodic repolarization dynamics: MADIT-II substudy." European heart journal, vol. 294, pp. 1240-1247, 2017.

[48] M. C. Haigney, W. Zareba, P. J. Gentlesk, R. E. Goldstein, M. Illovsky, S. McNitt, M. L. Andrews, and A. J. Moss, "QT interval variability and spontaneous ventricular tachycardia or fibrillation in the Multicenter Automatic Defibrillator Implantation Trial (MADIT) II patients," J Am Coll Cardiol, vol. 44, no. 7, pp. 1481-1487, 2004.

[49] A. Porta, V. Bari, F. Badilini, E. Tobaldini, T. Gnecchi-Ruscone, and N. Montano, "Frequency domain assessment of the coupling strength between ventricular repolarization duration and heart period during graded head-up tilt," J. Electrocardiol., vol. 44, no. 6, pp. 662-668, 2011.

[50] M. Baumert, M. P. Schlaich, E. Nalivaiko, E. Lambert, C. I. Sari, D. M. Kaye, M. D. Elser, P. Sanders, and G. Lambert, "Relation between QT interval variability and cardiac sympathetic activity in hypertension," $\mathrm{Am}$ J Physiol Heart Circ Physiol, vol. 300, no. 4, pp. H1412-H1417, 2011.

[51] S. Nayyar, K. C. Roberts-Thomson, M. A. Hasan, T. Sullivan, J. Harrington, P. Sanders, and M. Baumert, "Autonomic modulation of repolarization instability in patients with heart failure prone to ventricular tachycardia," Am J Physiol Heart Circ Physiol, vol. 305, no. 8, pp. H1181-H1188, 2013.

[52] R. Furlan, A. Porta, F. Costa, J. Tank, L. Baker, R. Schiavi, D. Robertson, A. Malliani, and R. Mosqueda-Garcia, "Oscillatory patterns in sympathetic neural discharge and cardiovascular variables during orthostatic stimulus," Circulation, vol. 101, no. 8, pp. 886-892, 2000.

[53] P. Taggart, P. Sutton, Z. Chalabi, M. R. Boyett, R. Simon, D. Elliott, and J. S. Gill, "Effect of adrenergic stimulation on action potential duration restitution in humans." Circulation, vol. 107, no. 2, pp. 285-289, 2003.

[54] K. Fukuda, H. Kanazawa, Y. Aizawa, J. L. Ardell, and K. Shivkumar, "Cardiac innervation and sudden cardiac death," Circ. Res., vol. 116, no. 12 , pp. 2005-2019, 2015.

[55] M. Noriega, J. P. Martínez, P. Laguna, R. Bailón, and R. Almeida, "Respiration Effect on Wavelet-Based ECG T-Wave End Delineation Strategies," IEEE Trans. Biomed. Eng., vol. 59, no. 7, pp. 1818-1828, jul 2012.

[56] G. Valenza, M. Orini, L. Citi, A. Minchole, E. Pueyo, P. Laguna, and R. Barbieri, "Assessing instantaneous QT variability dynamics within a point-process nonlinear framework," in 2014 8th Conference of the European Study Group on Cardiovascular Oscillations (ESGCO). IEEE, 2014, pp. 67-68. 